UNIVERSITAS, Volumen 4, Número 1, 2013, 26-28, ISSN 2071-2574

(c) UNAN-León, Editorial Universitaria

\title{
EVALUACIÓN DEL COMPONENTE CURRICULAR DE SOCIOLOGÍA QUE SE REALIZÓ EN EL PERÍODO 2007-2008 EN LA UNAN - LEÓN, COMO INSUMO PARA LA MEJORA CONTINUA.
}

\author{
Xochilt González Gutiérrez. Departamento de Ciencias Sociales, Facultad de Ciencias de la \\ Educación y Humanidades, Universidad Nacional Autónoma de Nicaragua, León (UNAN-León).
}

\section{RESUMEN}

El presente artículo tiene como finalidad mostrar una propuesta mejorada del programa curricular de Sociología que se impartió en el período 2007 - 2008. Para su realización se evaluaron los elementos del programa, como contenidos, metodologías de enseñanza y evaluación del aprendizaje; teniendo en cuenta la opinión de diferentes fuentes involucradas: estudiantes, docentes y expertos de UNAN - León y Universidad Centroamericana (UCA). La información se obtuvo a través de encuestas aplicada a estudiantes, entrevistas a profesores que impartieron el componente curricular y expertos en el campo de la Sociología. Se encontró que de los 23 temas abordados solamente $60.87 \%$ fueron considerados importantes por los estudiantes; en cambio los profesores y expertos los valoraron como apropiados. A juicio de las fuentes consultadas las metodologías de enseñanza necesitan tener mayor coherencia con los contenidos y que en la evaluación del aprendizaje las habilidades que presentaron mayores dificultades son la búsqueda bibliográfica (56.8\%) y la comprensión del nuevo conocimiento (56.1\%).

Palabras Claves: Sociología, evaluación curricular, mejora continua.

\section{INTRODUCCIÓN}

La evaluación curricular, se define como el proceso mediante el cual se recoge e interpreta, formal y sistemáticamente, información pertinente sobre un componente curricular, se producen juicios de valor $\mathrm{y}$ se toman decisiones para mantener, proyectar, reformar o eliminar elementos, que impidan alcanzar las competencias definidas ${ }^{[1]}$.

La Reforma Académica iniciada en la UNAN-León en 1995, introdujo cambios en el currículo, que incluía: modelo educativo, contenidos, formas de enseñanza y evaluación del aprendizaje; con el fin de modernizar y hacer más pertinente el proceso dentro de una cultura de evaluación y de mejora continua ${ }^{[2] .}$

En el 2007, en el marco del proceso de mejora continua, se incorpora en el II semestre del Año de Estudios Generales, el componente de Sociología y en el 2009, teniendo en cuenta la necesidad de reflexionar sobre lo que se ha hecho, se consideró pertinente realizar una evaluaciónal componenteque de forma objetiva indicara los cambios que se deben realizar. Dicha evaluación partió de inquietudes, tales como: ¿La Sociología contribuye a formar los profesionales integrales que demanda el país? ¿Los elementos del currículo definidos en el programa de Sociología responden a las necesidades del perfil del Semestre de Estudios Generales? ${ }^{[3,4,5] \text {. }}$

Es en este esfuerzo, que se enmarca el presente trabajo y ofrece un espacio de reflexión sobre la práctica educativa de los diferentes elementos del programa de Sociología y a la luz de las lecciones aprendidas, propone las mejoras necesarias para un mejor cumplimiento de sus competencias.

Este trabajo además puede servir de referente para continuar perfeccionando el proceso de mejora del Programa de Sociología y de los diferentes componentes curriculares del Semestre de Estudios Generales. ${ }^{[4 \text { y } 5]}$ 


\section{DISEÑO METODOLÓGICO}

El estudio es exploratorio, descriptivo, $\mathrm{La}$ muestra se conformó con 4 estudiantes de cada uno de los 33 grupos, para un total de 132. El número de académicos seleccionado fue 11 de un total de 21. El total de la muestra de expertos fue seis, que por sus características académicas y profesionales se consideraron idóneos para brindar información que le diera mayor fortaleza al análisis de los resultados.

A los estudiantes se les aplicó una encuesta, utilizando el formato de la Escala Likert. ${ }^{[6]} \mathrm{A}$ los académicos y expertos se les realizó una entrevista con preguntas abierta, obteniéndose resultados cuali-cuantitativos.

Para todos los grupos se formularon preguntas relacionadas con las siguientes variables: contenidos, metodología de enseñanza y evaluación del aprendizaje; así como la correspondencia entre dichos elementos.

Con la información obtenida se valoraron los puntos coincidentes y en los que no había acuerdo se analizaron sus posibles causas para llegar a conclusiones más justas e imparciales.

\section{RESULTADOS Y DISCUSIÓN}

Los estudiantes consideraron que de los 23 temas abordados, el $60.8 \%$ son importantes para su cultura general. Los académicos y expertos en términos generales los valoraron como apropiados; sin embargo, opinan que se necesita agregar temáticas afines con la problemática actual.

La coherencia entre contenidos y metodologías, muestra un grado de insatisfacción del $60.6 \%$ para los estudiantes. En términos generales los académicos opinaron que debido a dificultades de tiempo, complejidad y nivel de abstracción de algunos contenidos no cumplieron con el $100 \%$ de las metodologías orientadas en la planificación docente. Los expertos reconocieron la importancia de utilizar diferentes metodologías de enseñanza, teniendo en cuenta el tipo de contenido y las competencias a desarrollar.

De acuerdo a opinión de los estudiantes, el sistema de evaluación en correspondencia con la metodología, muestra un mayor grado de satisfacción de $58 \%$, lo que puede entenderse por las diversas formas en que se realizan los trabajos grupales, informes y exposiciones. El 100\% de los docentes opinan que la evaluación refleja vacíos que deben ser superados, como el asignarle mayor valor a las evaluaciones parciales y el tipo de pregunta que no favorece el análisis y la reflexión. Los expertos coinciden en la importancia de la evaluación en el proceso de aprendizaje y que el tipo de pregunta debe medir la compresión y la aplicación del conocimiento en contextos diferentes al aula.

\section{CONCLUSIONES}

Poca experiencia en el diseño, organización, ejecución y evaluación de programas curriculares basados en competencias.

Los contenidos en términos generales están bien, pero es necesario dosificar el tiempo asignado y contextualizarlos para lograr aprendizaje significativo.

Débil correspondencia entre las metodologías implementadas y los contenidos desarrollados.

Poca experiencia en la aplicación de la evaluación como proceso formativo, ya que el tipo de preguntas de la evaluación escrita favorece el desarrollo de las habilidades memorísticas y la práctica de la retroalimentación es insuficiente.

Se ratificó que el componente de Sociología es importante para desarrollar capacidad de análisis y de reflexión crítica de los fenómenos sociales, económicos y culturales de la sociedad contemporánea. 


\section{AGRADECIMIENTOS}

- M.Sc. Vinicio Sandino.

- M.Sc. Olivia Gutiérrez Morales

\section{REFERENCIAS}

1. Nolla, N;(2004), "Instrumentos para la evaluación y certificación del diseño curricular". Escuela Nacional de Salud Pública, Habana.vol. 18 No.3

2. M.A. Santos Guerra (1993). Los abusos de la evaluación: In Santos Guerra. la evaluación, un proceso de diálogo, comprensión y Mejora. Ed. Aljibe Archidonna.

3. Proyecto Educativo UNAN - León. 1997.

4. Tunnermann, Berheim, C; (2001). "La Educación Superior y los desafíos del Siglo XXI”. Managua, Nicaragua.

5. Conferencia Mundial sobre la Educación Superior en el siglo XXI": Visión y Acción. UNESCO. París. 1998.

6. Blanco, N; y Alvarado, M. E; (2005) "Escala de Actitud hacia el Progreso de Investigación Científico Social". Revista de Ciencias Sociales, sep., vol.11, No.3, pág. (537- 546). 